\title{
Celebrando 80 años al servicio de la Salud Integral de la Mujer: invitación abierta a ser parte de un congreso que enaltece el rol histórico del especialista
}

Este año nuestra sociedad cumple 80 años. Desde su creación, su objetivo central ha sido el contribuir a garantizar los mejores estándares de atención en salud para la mujer. De esa forma, ginecólogos obstetras y matronas del pasado, presente y futuro, han contribuido y contribuirán a mejorar sostenidamente los indicadores de salud y a mejorar las políticas que rigen en nuestro país.

A fin de mantener dicha premisa, cada dos años, los miembros del directorio de nuestra sociedad aunaron sus esfuerzos en pos de levantar un Congreso que garantizara la educación continua. Inicialmente limitado en recursos y número de asistentes, década tras década, se fue transformando en un evento esperado y en el cual todos los participantes esperaban adquirir un nuevo conocimiento o herramienta a aplicar. Por pequeña que ésta fuese, sería útil en tratar y salvar la vida de la próxima paciente que nos tocara ver, ese es el norte que a todos nos motiva.

Es por ello, que este año no puede ser la excepción. Estamos ad portas de concretar la XXXV versión de nuestro Congreso bienal de la especialidad y la tarea no ha sido fácil. Para el directorio en ejercicio, ha sido su deber el buscar un temario que refleje las necesidades históricas y actuales de nuestra sociedad. Así también ha sido un desafío el sortear las estrecheces económicas que los cambios legales han introducido en la relación sociedad-empresa y el estado actual de la economía nacional. Esto no ha de ser visto como una excusa sino como una fuente de motivación para buscar el mejor resultado, centrado en privilegiar la academia y la educación continua y garantizar la participación activa de todos aquellos que harán el esfuerzo de asistir y de aprender.

En primer lugar hemos decidido mantener el lugar habitualmente sede, esto es Viña del Mar. Un lugar donde se puede respirar la tranquilidad de la brisa marina y hacer una parada en el ajetreo diario

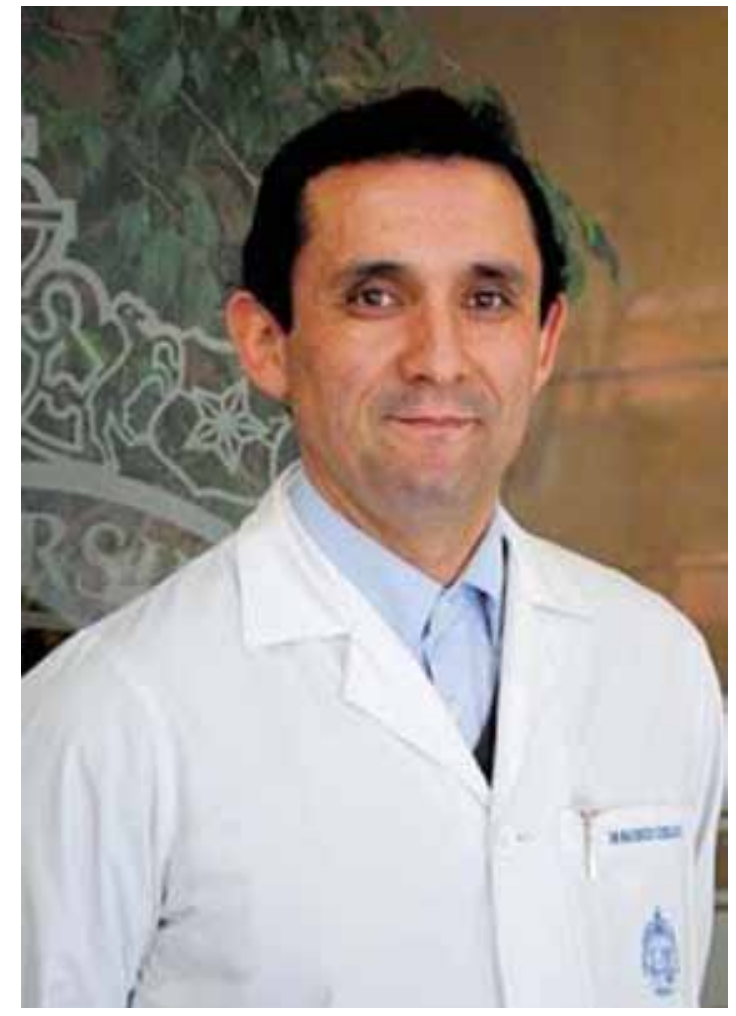

de nuestra especialidad. Sus vistas, los lugares, las entretenciones, ofrecen el complemento lúdico ideal para un esfuerzo académico. A diferencia de jornadas anteriores, hemos decidido concentrar las actividades lectivas en un solo hotel, el Hotel Enjoy Viña del Mar Casino \& Resort y en sólo dos días y medio. La fecha será entre el 11 y 13 de Noviembre del 2015. 
Para cumplir las expectativas, durante el año hemos preguntado tanto en Santiago como en reuniones realizadas en provincia, ¿cuáles son los temas que debemos privilegiar o abordar por relevancia? Hemos escuchado, visto y acogido los intereses de pares, de matronas y de la comunidad, y en base a ello hemos hecho modificaciones sustantivas no solo en el temario escogido sino a la modalidad de ejecución de cada una de las actividades. Todo ello con el fin de responder a las necesidades individuales, a las del país, de facilitar la participación, de ser inclusivos y de que todos puedan aprovechar al máximo la experiencia.

El primero de los días, solo en la tarde, estará destinado a cursos pre-congreso, de ejecución simultánea, tocando áreas temáticas de diverso interés. Los otros dos días, de jornada completa, se ocuparán con charlas plenarias, dictadas en su mayoría por reconocidos expertos internacionales, presentación de trabajos nacionales seleccionados (en modalidad póster $u$ oral), reuniones almuerzo temáticas y una actividad interactiva conocida como 'desafiando al maestro' organizada por los 'junior fellows'. Todo ello combinado con espacios para el café de interacción entre pares y conocimiento de los productos de interés presentados por las empresas patrocinantes.

La temática será diversa y abordara los problemas de salud que creemos más relevantes para el hoy. Todos tendrán la oportunidad de escuchar sobre patologías que comprometen la salud de la mujer a lo largo de toda la vida. Ello como reflejo de nuestra tradición histórica: 80 años al servicio de la Salud Integral de la Mujer. A modo de ejemplo hoy nos convoca la discusión abierta sobre la despenalización del aborto frente a tres causales. Es por ello que abordaremos áreas relacionadas que desde lo técnico garanticen el manejo correcto de esta problemática. Hemos preparado un taller de actualización y una conferencia plenaria sobre el manejo seguro del aborto. Así conoceremos del uso de la aspiración manual endouterina (AMEU), una forma costo efectiva de manejar el aborto incompleto y retenido. Así también, abordaremos el diagnóstico antenatal ultrasonográfico de malformaciones fetales a través de un curso pre-congreso y las alternativas de corrección de algunas de ellas en la vida intrauterina.

Desde la prevención abordaremos el rol de los nuevos métodos diagnósticos para el tamizaje de la patología cervical.

En el ámbito de la reproducción, abordaremos el manejo actual de la endometriosis. Junto a ello hemos considerado relevante el abordar el rol de la obesidad en problemas reproductivos, incluyendo síndrome metabólico y de ovario poliquístico.
También abordaremos de manera integral los problemas de la fertilidad que afectan al hombre. Para ello habrá un taller almuerzo referente a andrología para el ginecólogo obstetra.

En Ginecología abordaremos problemas prevalentes para la mujer postmenopáusica, incluyendo el enfrentamiento diagnóstico y terapéutico de la incontinencia urinaria, la patología mamaria y el manejo actual e integral del climaterio y menopausia. Para ello tendremos plenarias, cursos pre-congreso y almuerzos temáticos. En esta área hemos invitado también aun destacado patólogo quien revisará los avances en la etiopatogenia del cáncer de ovario y nuevas técnicas diagnósticas en patología ginecológica. Finalmente hemos abierto un espacio para la comunicación de videos quirúrgicos de interés clínico.

En el ámbito obstétrico un foco relevante será el curso pre-congreso sobre urgencias obstétricas, que esperamos cuente con la participación de médicos que hacen turno y residentes en formación.

No hemos querido dejar fuera la administración en Salud. Para ello hemos solicitado la participación de docentes nacionales quienes nos harán una puesta al día desde la perspectiva atingente a nuestra especialidad.

Junto a todos estos temas, también se abordarán aspectos de la ginecología infanto-juvenil, de la relación médico paciente, del devenir de la especialidad en los próximos 10 años, de la importancia del trabajo en equipo, y muchos otros temas más.

Otra actividad relevante para esta ocasión será el reconocimiento a dos maestros nacionales de la especialidad y a ginecólogos obstetras que por años, y tal vez anónimamente, han servido a la mujer chilena desde Arica a Punta Arenas.

En suma, creemos será una experiencia enriquecedora no sólo en el plano académico sino en lo humano y relacional.

Dado que la recertificación es una tarea de todos, hemos dispuesto que exista control de asistencia, un número suficientes de horas lectivas, el abordaje de temas de relevancia y una breve evaluación-encuesta a fin de cumplir con la normativa establecida por la Comisión Nacional de Certificación de Especialidades Médicas (CONACEM) y así otorgar el mayor creditaje posible a cada uno de los asistentes. Es deber de cada uno el cumplir con las exigencias y completar los registros de asistencia y la evaluación a fin de recibir el certificado con sus créditos respectivos.

Finalmente queremos resaltar que considerando que es nuestro deseo que la Sociedad comprenda a todos los especialistas en ejercicio, hemos dispuesto tarifas especiales y preferenciales a todos aquellos médicos que se inscriban con antelación al evento. 
Así también hemos dispuesto descuentos adicionales del cual serán beneficiarios todos aquellos que hayan recibido el reconocimiento de su membresía y pagado las cuotas antes del 1ํ de Octubre del 2015.

Así, la invitación ya está hecha. Los esperamos con sus contribuciones al congreso, con sus ganas de aprender y de disfrutar de un ambiente grato y de camaradería. Viña del Mar y la Sociedad, presentes para mantener nuestra esencia.... Siempre al servicio de la Salud Integral de la Mujer.

Dr. Mauricio Cuello Fredes Presidente Sociedad Chilena de Obstetricia y Ginecología 